Rev. Elev. Méd. vét. Pays trop., 1978, 31 (3) : 281-286.

\title{
La Maladie Nodulaire Cutanée des Bovins en Côte-d'Ivoire
}

\author{
par F. PIERRE (*)
}

\begin{abstract}
RESUMÉ
L'auteur s1gnale l'apparition, pour la première fois, en Côte d'Ivoire, de la maladie nodulaire cutanée des bovins (M. N. C. B.) sans qu'il lui soit possible d'en situer l'origine.

Il en décrit la symptomatologie, l'évolution et l'épidémiologie en précisant qu'il a observé une forme miliaire, confirmée par le laboratoire, forme qui ne paraît pas avoir été décrıte antérieurement.

La maladie qui dans la plupart des cas guérit spontanément peut parfois s'accompagner de complications dues à des infections secondaires plus ou moins graves.
\end{abstract}

\section{INTRODUCTION}

C'est le 21 janvier 1976, lors d'une visite technique, que le Dr Vétérinaire J. CHAMBRON, Directeur de projet et moi-même effectuons au Centre de recherches Zootechniques à Bouaké, que nous fut signalée une affection à allure contagieuse frappant dans de notables proportions $(25-60$ p. 100) la totalité des troupeaux de bovins N'Dama et Jersiais de la ferme. Elle se caractérise alors par l'apparition de nombreux nodules cutanés de différentes dimensions envahissant tout le corps des animaux, accompagnés d'une violente réaction des ganglions lymphatiques et dans certains cas d'un état de déchéance accusée nécessitant l'abattage de quelques-uns.

L'hypothèse de la maladie nodulaire cutanée des bovins a été retenue et s'est trouvée confirmée par le Laboratoire de Dakar-Hann sur les échantillons qui y ont été acheminés à des fins diagnostiques.

(*) Dr Vétérinaire, expert F. A. O./P. N. U. D. au projet IVC/71/505, Laboratoire de pathologie animale de Bingerville, responsable du laboratoire annexe de Korhogo, B. P. 32, Côte-D'Ivoire.

\section{II. ÉPIZOOTIOLOGIE}

$\mathrm{La}$ découverte et l'identification de la M. N. C. B. est restée localisée à Bouaké jusqu'au 11 novembre 1976, date à laquelle nous avons été alertés par la progression rapide et envahissante d'une semblable affection autour de la ville de Korhogo. La visite d'une dizaine de parcs dans les villages de Gbonkaha, de Koni, de Nithion et de Fodonithion a permis de reconnaître et de confirmer la présence de la M. N. C. B. dans le nord du pays. Les renseignements recueillis auprès des éleveurs peulhs sont formels : la maladie est d'apparition récente et inconnue dans le milieu (5).

La nouvelle affection est donc apparue fin saison de pluie, début saison sèche. Elle s'est répandue en tache d'huile autour de Korhogo en suivant un axe nord-nord est, depuis Fodonithion, Nithion, Taouara, Kolévogo, Fonomvogo, Lataha, Sinématiali jusqu'à Ferkessédou gou, puis bifurque tout à fait au sud de Korhogo dans toute la région de Napiéolédougou jusqu'à Kiémou. Elle a sévi durant tout le mois de janvier à l'intérieur du triangle délimité par les 3 sommets suivants : Fodonithion, Ferkessédougou et Kiémou (carte). 


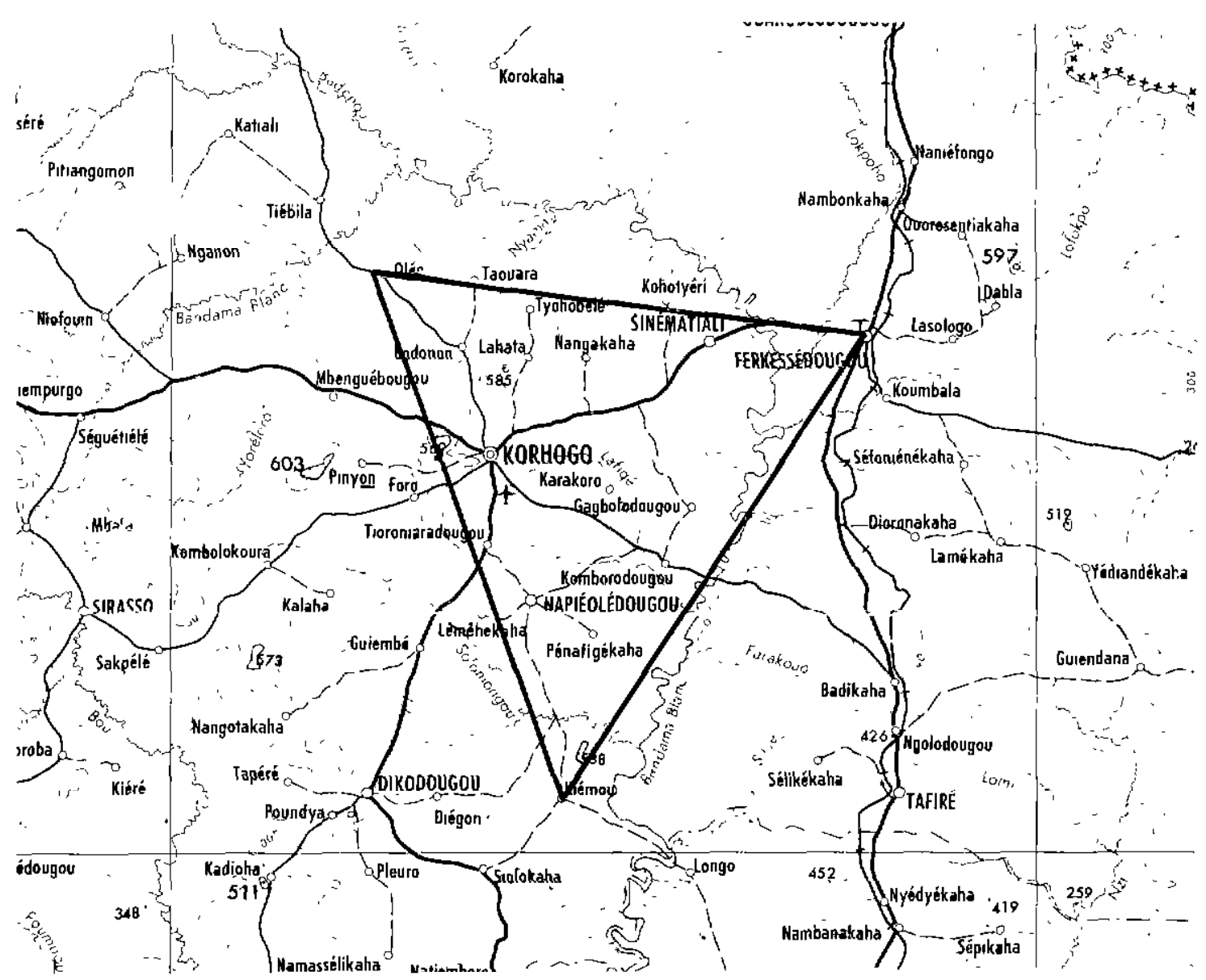

Plus au nord, la maladie a été diagnostiquée le 26 novembre 1976, sévissant de façon très circonscrite dans quelques 2 parcs très retirés à Tengrela.

En 1977 , le M. N. C. B. n'est pas réapparue dans les anciens parcs atteints. Par contre, elle a surgi dans le Secteur Sud-Ouest de Korhogo sur l'axe, Foro, Dassoungbo, Kombolokoura, etc... et a disparu aussi brusquement sans faire trop de dégât à la fin du mois de décembre.

La présence de la M. N. C. B. à Tengrela, c'est-à-dire à quelque $600 \mathrm{~km}$ de Bouaké et à $300 \mathrm{~km}$ de Korhogo, son second point d'apparition, pose le problème de son berceau d'origine. La chronologie de nos observations n'en facilite guère l'explication.

L'affection a été découverte par hasard à Bouaké. A-t-elle émigré de la ferme à quelques parcs du nord ? Cela expliquerait, certes, l'irruption de la maladie à Korhogo sans répondre à l'énigme de sa présence même à Bouaké. Par contre, sa découverte à Tengrela donne plus de vraisemblance à une arrivée probable par cette porte d'entrée des animaux en Côte-d'Ivoire, et une contamination linéaire progressivement descendante sur Korhogo et environs, puis sur Bouaké, axe naturel des migrations animales jusqu'à Abidjan. Mais l'existence de la M. N. C. B. n'a pas été signalée dans le sud du pays. Dans ces conditions, la porte d'entrée, les voies de cheminement ainsi que le mode de transmission naturelle de la maladie demeurent pour nous hypothétiques.

Il est d'abord frappant de constater que ce sont les animaux très infestés par des tiques qui portent le plus grand nombre de nodules. Ceux-ci semblent confluer dans les régions du corps les plus endommagées par ces parasites.

Ensuite l'extension de la M. B. C. B. semble procéder de proche en proche, par voie de contiguïté et peut-être aussi par bonds (4), de troupeau à troupeau, d'animal sensible à animal sensible (6). Cette extension est favorisée par tout un réseau de liaisons inextricables ajoutant leurs effets les uns aux autres lors de déplacements à travers les savanes herbeuses aux sources communes d'abreuvement au cours desquels les animaux sont soumis aux agressions de hordes 
d'insectes piqueurs (glossines, taons, moustiques... etc.), agents probables facilitant le passage du virus d'animal à animal.

Enfin la M. N. C. B. est concomitante dans certains parcs de quelques autres affections dont il faut la différencier et dans l'étiologie desquelles on suspecte l'intervention de vecteurs vivants.

Tous les bovins indistinctement peuvent en être atteints quelles que soient la race ou la couleur : taurins ou zébus, N'Dama, Baoulé ou Jersiaise ; et enfin l'âge ou le sexe.

Malgré son allure explosive et contagieuse, la M. N. C. B. ne frappe pas plus que 20 à 40 p. 100 de l'effectif des troupeaux ; la mortalité naturelle est faible sinon nulle.

\section{SYMPTOMATOLOGIE}

\section{L'affection est protéiforme à son début.}

Certains animaux commencent par présenter de l'inappétence ; d'autres émettent une abondante salivation, du jetage séro-muqueux ou muco-purulent. La plupart sont gênés par un larmoiement séreux ou purulent qui colle et ferme les paupières : il s'agit d'une conjonctivite, voire d'une kératite qui retient d'abord l'attention des éleveurs. Ils sont ensuite inquiétés par l'apparition soudaine de nodules qui "poussent manifestement à l'ceil» sur tout le corps des animaux. En moins d'une journée, les plus gros atteignent leur taille définitive, 3 à $4 \mathrm{~cm}$ de diamètre. Ils se localisent plus particulièrement à l'encolure, au fanon, sur les côtés, à la face postéro-interne des cuisses, au périnée, sur les mamelles, au creux des flancs. Ces nodules sont fermes, indolores, franchement implantés dans le derme profond, adhèrent fortement à la peau, suivent ses mouvements et en conservent les attributs (couleur, poils, etc...).

D'autres animaux, surtout des veaux, montrent d'énormes nodules douloureux de par leur position sur les muqueuses nasales et buccales. Ils les empêchent littéralement de respirer, de têter ou de manger avec risque évident d'asphyxie ou de diète prolongée.

Une forme qu'on pourrait qualifier de miliaire coexiste avec les aspects précédents. Elle apparaît avec une rare intensité sur les animaux à peau tendre, les jeunes et les zébus. Elle se caractérise par la présence d'une multitude de petits nodules de la grosseur d'un grain de mil à celle d'un petit pois. Ils confluent sur la face latérale de l'encolure, aux confins du périnée. Ces régions sont comme parcheminées et laissent au toucher une sensation granuleuse.

Sur les animaux intensément maculés de nodules, de violentes réactions ganglionnaires se manifestent au niveau des préscapulaires, des cruraux et des poplités. Ce sont des masses tumorales fermes de $8,12,18 \mathrm{~cm}$ de diamètre qui s'ulcèrent et s'infectent, laissant des cavités lentes à se combler et à se cicatriser.

Dans les parties déclives du corps, l'affection progresse sur certains animaux par un œdème envahissant qui gonfle les membres, provoque des boiteries douloureuses, gêne la marche et force finalement au décubitus. $\mathrm{Ca}$ et là, au genou, au boulet, à la couronne (Photo 1), des zones de nécrose s'ouvrent, laissant béantes des plaies ulcéreuses, circulaires de 4 à $7 \mathrm{~cm}$ de diamètre, profondes, à bords tourmentés, d'où il sourd par simple pression du pus granuleux.

\section{Lésions}

Les animaux autopsiés sont morts soit de mort naturelle par suite de décubitus et de dic̀tc prolongés, soit par abattage, vu l'état de déchéance de certains malades.

Les lésions se limitent aux membres œdémaciés. Ceux-ci montrent de nombreuses fistules draînant de multiples abcès. Il en sort, après incision, du pus jaune citrine bien lié. A la culture bactériologique, il en est sorti des germes de complication : Cocci, Corynébactérium... etc.

Aucune lésion pathognomonique n'a pu être mise en évidence dans les voies digestives ou sur les viscères.

Les symptômes et les lésions se manifestent en tout ou partie sur les atteints.

\section{Evolution}

Les nodules de taille moyenne s'éliminent progressivement sous forme de croûtes sèches, après disparition rapide des poils. L'architecture du derme recouvre peu à peu ses attributs et sa fonction (6).

Les plus gros nodules, l'œdème des membres et l'inflammation des ganglions s'ouvrent par une fistule qui creuse bientôt une plaie cavitaire. 


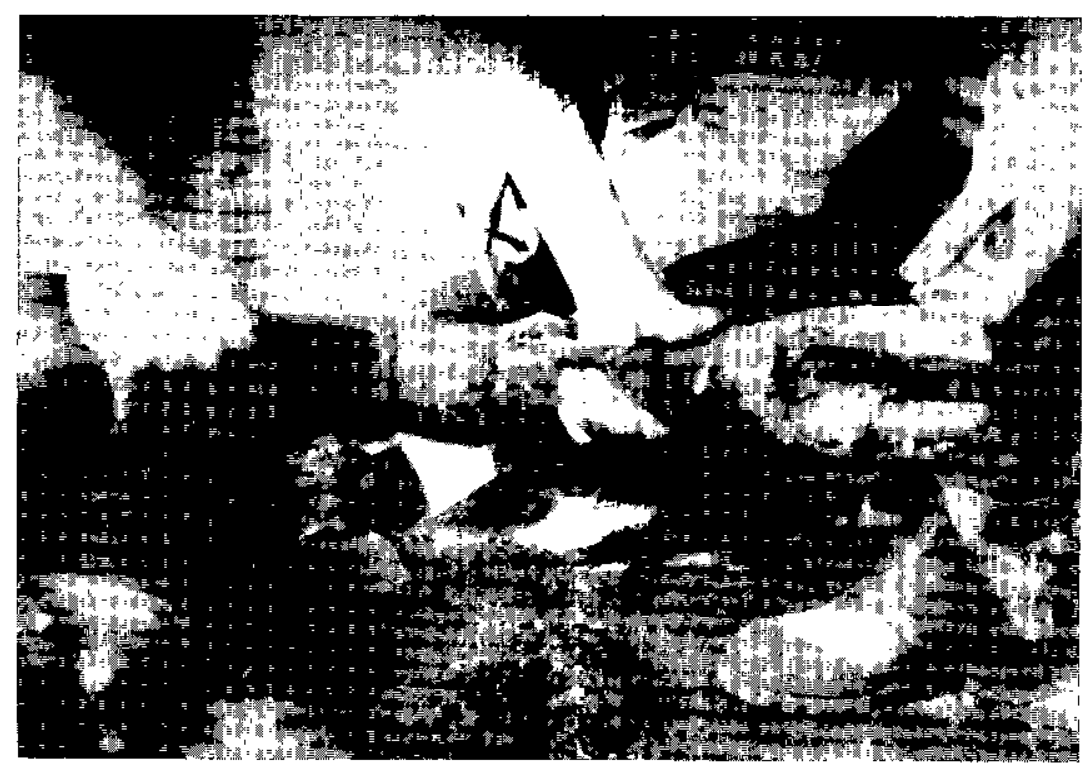

Photo 1. - Membre cedémacié d'un bovin baoulé avec fistules et plaie ulcéreuse.

Photo 2. - Veau N'dama littéralement couvert de nodules en voie de cicatrisation.

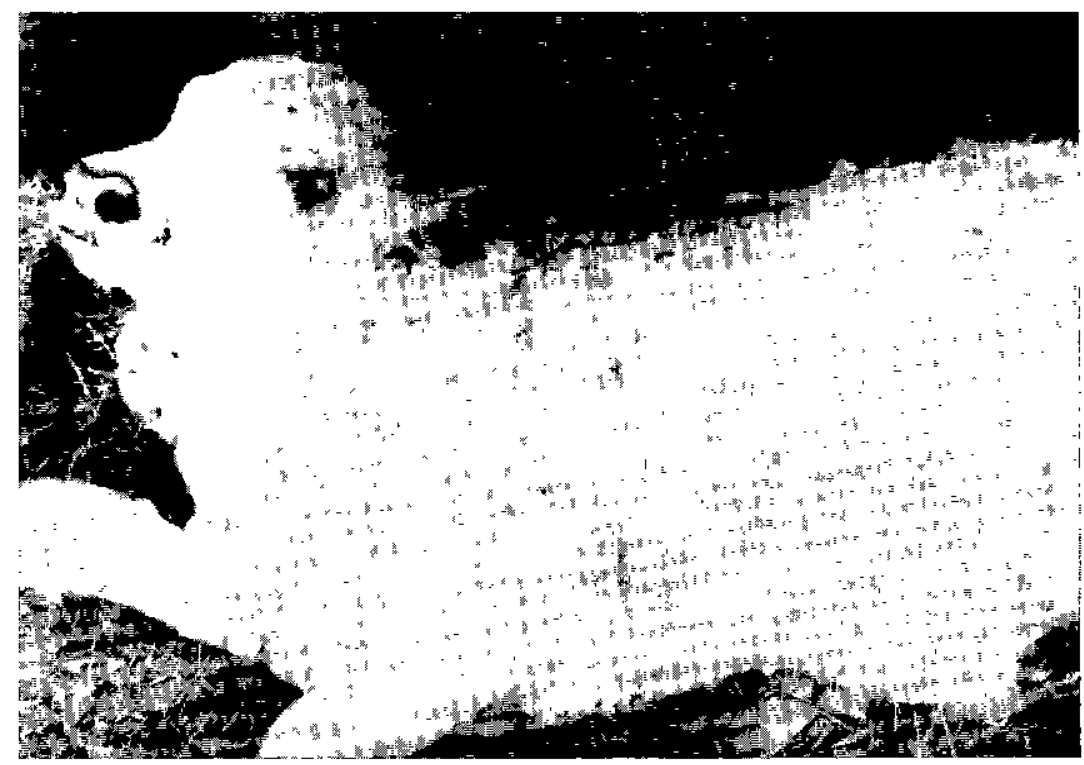

Celle-ci se cicatrise lentement ou s'infecte, suppure, conduit parfois à une érosion voire une destruction du tissu osseux.

L'état général peut être diversement atteint. Dans l'ensemble du troupeau, la M. N. C. B. régresse 1 à 2 mois après son apparition. Ce sont surtout les ulcères surinfectés qui traînent.

\section{Diagnostic}

Deux ans après son installation, la M. N. C. B. est identifiée dès qu'elle réapparaît dans la région nord.
D'abord, par sa symptomatologie typique des nodules qui ne prêtent guère à confusion avec d'autres maladies. Ensuite par son épizootiologie : cycle annuel de début de saison sèche; allure explosive, extension rapide, morbidité moyenne de 25 p. 100 et mortalité insignifiante.

Cependant, il y a lieu de la différencier de 2 affections couramment rencontrées dans la zone dense de Korhogo.

\section{Il s'agit}

1) de la démodexose, caractérisée par la présence sur tout le corps y compris la tête (pourtour des yeux, tout le chanfrein) de nodules 
ou de pseudo-nodules fistulisés d'où sourd par pression un tortillon de pus bourré de demodex. La peau est épaissie et plissée ; elle paraît comme crevassée et sale. Le jeune qui en est atteint présente un aspect sénile ;

2) la dermatophilose qui est plutôt une affection de la belle saison. C'est une dermatose suintante avec hérissement des poils qui se trouvent progressivement "noyés dans un exsudat coagulé » (1). Les lésions nodulaires sont superficielles. L'affection traîne longtemps dans les élevages.

\section{Pronostic}

En soi, le pronostic de la M. N. C. B. est bénin puisqu'elle n'atteint qu'une faible partie de l'effectif des troupeaux. Mais il peut devenir inquiétant et s'assombrir même quand le mal s'attaque aux veaux. La mort est possible et survient par inanition et épuisement (avec diarrhée profuse).

\section{Traitement}

La M. N. C. B. étant d'origine virale, la thérapeutique ne vise qu'à éviter les complications bactériennes. L'intervention avec des produits antiseptiques ou antibiotiques s'est révélée efficace.

\section{Prophylaxie}

Aucune mesure de prophylaxie médicale ou sanitaire ne se justifie dans l'immédiat, eu égard à l'insignifiance des pertes causées par la M. N. C. B.

\section{SUMMARY}

\section{Bovine lumpy skin disease in Ivory Coast}

Bovine lumpy skin disease was diagnosed in the farm of the « Centre de Recherches Zootechniques » at Bouaké-Minankro in January and at Korhogo and Tengrela during November 1976. The informations collected together with the symptoms observed led us to suppose that this was the first appearance of the disease on these farms. Bovine lumpy skin disease affects cattle without distinction as to age, sex, colour and breed (N'dama, Baoulé, Zebu, Jersey) but has a predisposition for animals with finer skin.

The disease is characterised by the presence of numerous cutaneous nodules on the body particularly in areas of loose skin with cedematous swellings in the dependent areas and on the lymphatics. These symptoms are accompanied by conjunctivitis or kerato-conjunctivitis and the appearance of ulcers on the limbs. Rarely a nodule bursts and changes into an ulcer but cedema favours necrosis of the affected parts which rupture and become contaminated leading the animals to become lame and to remain lie down.

This disease is benign in nature and some two months after its appearance the lesions begin to decrease gradually and disappear.

Symptomatic treatment with antiseptics and antıbiotics is effective but prophylaxis is not necessary for the present.

\section{RESLMEN}

\section{La dermatosis nodular de los bovinos en Costa de Marfil}

Se identifica la dermatosis nodular de los bovinos por primera vez en la granja del Centro de Investigaciones zootecnicas de Buake-Minankro, en enero de 1976. Se la encuentra tambien en noviembre del mismo año en Korhogo y en Tengrela. Según las informaciones recogidas, es la primera vez.

Ataca indiferentemente bovinos o cebues de cualquiera raza y de cualquiera color, Ndama, Baule, Jersey, de cualquier edad y de cualquier sexo, pero más particularmente los animales con piel tierna.

Se caracteriza por la presencia sobre el cuerpo de numerosos nódulos adheriendo a la piel en las regiones con tejido flojo, la edema en las partes en pendiente $\mathrm{y}$ reacciones ganglionares. Todo eso puede acompañarse por una conjuntivitis o una queratoconjuntıvitis y úlceras apareciendo al nivel de los miembros.

Los nodulos rara vez se ulceran pero las edemas de los miembros favorecen necrosis que se abren y se infectan, de dónde cojera con decúbito.

La afección es benigna, progresivamente desaparece espontáneamente unos dos meses después de su aparición.

El tratamiento sintomatico es eficaz con antisepticos y antibioticos. Por ahora no se justifica la profilaxis sin efecto. 


\section{BIBLIOGRAPHIE}

1. BOURDIN (P.). La maladie nodularre cutanée des bovidés. Paris, L'expansion scientifique, 1970.

2. BUCK (G.), QUENEL (J. J.); SERRES (H.). Une maladie nouvellement identifiée à Madagascar: la lumpy skin disease Rev. Elev. Méd, vét. Pays. trop., 1956, 9 (3) : 229-235.

3. DIAS (A. de S.), LIMPO-SERRA (J.). La dermatose nodulaire (Lumpy skin disease) au Mozambique. Bull. Off. int. Epiz. 1957, $46: 612-625$.
4. LALANNE (A.). La maladie nodulaire de la peau des bovins (Lumpy skin disease) à Madagascar en 1956 et 1957. Bull. Off. int. Epiz., 1957, 46: 596-611.

5. PIERRE (F.). Rapport annuel d'activité du laboratoire annexe de Korhogo pour l'année 1976. 1977, $23 \mathrm{p}$.

6. WEISS (K. E.). Dermatose nodulaire cutanée, maladie nouvelle des animaux. Rome, F. A. O., 1964, p. 179201. (Etude agricole F. A. O. n 61). 